\title{
CORRESPONDENCE
}

\section{The Range and Scientific Value of Randomized Trials}

Part 24 of a Series on Evaluation of Scientific Publications

by PD Dr. med. Stefan Lange, PD Dr. med. Stefan Sauerland, Dr. med. Dipl.-Psych. Jörg Lauterberg, and Prof. Dr. med. Jürgen Windeler in issue $38 / 2017$

\section{Central Problem is Equipoise}

Randomized controlled trials (RCTs) are the gold standard for clinical research, as the authors point out (1). Informed consent as well as consent to randomization are the ethical premises for most RCTs (2). Designs that completely eliminate informed consent are the low-risk pragmatic RCTs and the so-called prompted optional randomization trials (PORT).

A central problem in the ethics of clinical research is equipoise (3). Among other things, this dilemma explains why currently only $3-5 \%$ of potential patients are included in RCTs.

Interestingly, in the United States from 1973 to 2006 , 68 anticancer drugs were approved by the FDA without evidence from RCTs. A further problem in oncology are negative studies as well as phase II or phase III studies that were discontinued or failed. Indeed, from 2013 to $2015,32 \%$ of 5821 RCTs were discontinued or failed.

DOI: 10.3238/arztebl.2018.0114a

\section{References}

1. Lange S, Sauerland S, Lauterberg J, Windeler J: The range and scientific value of randomized trials-part 24 of a series on evaluation of scientific publications. Dtsch Arztebl Int 2017; 114: 635-40.

2. Grady C, Cummings SR, Rowbotham MC, McConnell MV, Ashley EA Kang G: Informed consent. N Engl J Med 2017; 376: 856-67.

3. Joffe S, Miller FG: Equipoise: asking the right questions for clinical trial design. Nat Rev Clin Oncol 2012: 9: 230-5.

Additional references available by request to the author.

Dr. med. Antonis G. Tsamaloukas

Berlin, Germany

tsamaloukas@oxphos.de

Conflict of interest statement

The author declares that no conflict of interest exists.

\section{No Substitute for a Doctor's Intuition}

„Randomized clinical trials (RCTs) are the gold standard for assessing relationships between intervention and outcomes", state the authors of the article (1). This statement is not entirely correct because it does not apply to the use of new antibiotics or vaccines. Antibiotics and vaccinations are clinically assessed based on their effects rather than on RCTs.

Clinically active physicians should not sacrifice their own judgment simply because a randomized study of certain treatments is lacking. Randomized studies can be very useful, for instance in chemotherapy; however, their use should be critically evaluated, and they should not replace medical observation and intuition.

DOI: 10.3238/arztebl.2018.0114b

\section{References}

1. Lange S, Sauerland S, Lauterberg J, Windeler J: The range and scientific value of randomized trials-part 24 of a series on evaluation of scientific publications. Dtsch Arztebl Int 2017; 114: 635-40.

\section{Dr. med. Elio Torremante}

Ochsenhausen, Germany

dr.torremante@onlinemed.de

Conflict of interest statement

The author declares that no conflict of interest exists.

\section{Real-World Usefulness Is Missing}

In the article by Lange et. al. (1), important aspects of real-world use are not mentioned. Randomized controlled trials (RCTs) are the gold standard for clinical trials in which the efficacy of an intervention under ideal conditions is examined. The primary goal of demonstrating efficacy is the answer to the question of evidence-based medicine: „Can it work?“ (1). The clarification of this question is classified as an experiment on humans and requires ideal conditions.

RCTs can no longer be required as a gold standard for clinical trials in which the effectiveness of an intervention under real-world conditions is examined. The primary goals for demonstrating effectiveness and fitness for real-world use relate to answering the questions ,does it work, and is it worth it?" (2). Examining these two properties requires real-world conditions and instruments other than proof of efficacy (3).

Real-world conditions include co-morbidities and their therapies, which are perceived by researchers as disturbing factors. However, both questions of „does it work?" and „is it worth it?" can only be answered considering real-world care.

Testing for effectiveness is essential. Disturbing factors influence care outcome positively or negatively. The lack of equipoise prevents patients from participating in RCTs. „Equipoise“ refers to the perceived comparability of two therapies, which - from the patients' point of view - should not be considered when, for example, the advantages and disadvantages of loss of a limb in surgery is weighed against radiotherapy for organ preservation.

As the correspondence format limits the number of arguments that can be presented, and the number of authors who can co-sign, we refer readers to our homepage www.clinical-economics.com for more information.

DOI: $10.3238 /$ arztebl.2018.0114c

\section{References}

1. Lange S, Sauerland S, Lauterberg J, Windeler J: The range and scientific value of randomized trials-part 24 of a series on evaluation of scientific publications. Dtsch Arztebl Int 2017; 114: 635-40. 
2. ke,Haynes B: Can it work? Does it work? Is it worth it? The testing of healthcare intervention is evolving. BMJ 1999; 319: 652-3. PMCID: PMC1116525.

3. Porzsolt F, Rocha NG, Toledo-Arruda AC, et al.: Efficacy and effectiveness trials have different goals, use different tools, and generate different messages. Pragmat Obs Res 2015; 6: 47-54.

Prof. Dr. med. Franz Porzsolt

President, Institute of Clinical Economics (ICE) e.V., Ulm, Germany mindset@clinical-economics.com

sekretariat@ice-ev.de

Prof. Dr. med. Karl-Walter Jauch

Head of Board, Hospital of the Ludwig-Maximilians-Universität München, Germany

\section{Conflict of interest statement}

The authors declare that no conflict of interest exists.

\section{In Reply:}

Porzsolt and Jauch highlight so-called, ,ideal conditions". It may be that narrowly defined conditions are necessary for certain issues. However, equating randomization and „ideal conditions" is fundamentally wrong. If one is even interested in the „real-world benefit" - and this idea is little more than a buzzword, then of course you can and should study this in an RCT. Nothing rules out using so-called pragmatic RCTs, as addressed in our publication (1). Tools other than RCTs are usually unnecessary, more elaborate, and (as everyone knows) provide less reliable results. In fact, only recently, authors of a study with so-called real-world data almost distanced themselves from their own results: despite a significant difference observed in favor of therapy escalation, they still were unwilling to base a recommendation on it (,real-life data are exposed to important potential biases"). (2). Nobody needs this (3).

Indeed, the main question is whether effects under the conditions described as ,ideal“ are different from others (effect modification). If one is interested in effect modification, then it would be highly unwise, for clarification in the context of another study, to change not only one factor for all study participants (,realworld" instead of ,ideal") but at the same time also a second one (non-RCT instead of RCT). In this case, differences in outcome in the two studies could not be attributed to any specific factor. Incidentally, evidence for obtaining significantly distinct effects in this way is surprisingly sparse.

Torremante rightly states that randomized trials should not replace medical observation and intuition. We would also like to emphasize his statement that one "s "own judgment" is required. However, the statement that RCTs play no role for antibiotics and vaccinations is not correct. Especially for vaccinations, drawing a conclusion about positive or negative effects due to ,clinical assessment“" is simply impossible. No one will be able to determine whether the fact that someone did not get the flu was due to being vaccinated or simply to not getting infected. The importance of using RCTs for vaccinations (for example, the HPV vaccine) as well as for antibiotics is self-evident, and it is equally evident that RCTs should be the basis of their assessment.

Tsamaloukas identifies equipoise as ,,a central problem in the ethics of clinical research". A call for equipoise as the most important ethical prerequisite is probably not helpful for the feasibility of RCTs, and if at all, then it should apply also to all other study types; we have addressed this in a further article that shall appear shortly in this journal.

DOI: 10.3238/arztebl.2018.0115

\section{References}

1. Lange S, Sauerland S, Lauterberg J, Windeler J: The range and scientific value of randomized trials-part 24 of a series on evaluation of scientific publications. Dtsch Arztebl Int 2017; 114: 635-40.

2. Delaloge $S$, Pérol D, Courtinard $C$, et al.: Paclitaxel plus bevacizumab or paclitaxel as first-line treatment for HER2-negative metastatic breast cancer in a multicenter national observational study. Ann Oncol 2016; 27: 1725-32.

3. Macleod MR, Michie S, Roberts I, et al.: Biomedical research: increasing value, reducing waste. Lancet $2014 ; 383$ : 101-4.

On behalf of the authors:

Dr. med. Dipl.-Psych. Jörg Lauterberg

IQWiG - Institute for Quality and Efficiency

in Health Care

Joerg.lauterberg@iqwig.de

Conflict of interest statement

The author declares that no conflict of interest exists. 